\title{
Collision-Free Mobile Robot navigation using Fuzzy Logic Approach
}

\author{
Mahmut Dirik \\ Department of Computer Engineering \\ Faculty of Engineering, Inonu University, Malatya, Turkey
}

\begin{abstract}
Autonomous mobile robots' navigation has become a very popular and interesting topic of computer science and robotics in the last decade. Many algorithms have been developed for robot motion control in an unknown (indoor/outdoor) and in various environments (static/dynamic). Fuzzy logic control techniques are an important algorithm developed for robot navigation problems. The aim of this research is to design and develop a fuzzy logic controller that enables the mobile robot to navigate to a target in an unknown environment, using WEBOTS commercial mobile robot simulation and MATLAB software. The algorithm is divided into two stages; In the first stage, the mobile robot was made to go to the goal, and in the second stage, obstacle avoidance was realized. Robot position information $(\mathrm{x}, \mathrm{y}, \varnothing)$ was used to move the robot to the target and six sensors data were used during the obstacle avoidance phase. The used mobile robot (E_PUCK) is equipped with 12 IR sensors to measure the distance to the obstacles. The fuzzy control system is composed of six inputs grouped in doubles which are left, front and right distance sensors two outputs which are the mobile robot's left and right wheel speeds. To check the simulation result for proposed methodology, WEBOTS simulator and MATLAB software were used. To modeling the environment in different complexity and design, this simulator was used. The experimental results have shown that the proposed architecture provides an efficient and flexible solution for autonomous mobile robots and the objective of this research has been successfully achieved. This research also indicated that WEBOT and MATLAB are suitable tools that could be used to develop and simulate mobile robot navigation system.
\end{abstract}

\section{General Terms}

Mobile Robot Control; Obstacle Avoidance, Fuzzy Logic Controller (FLC)

\section{Keywords}

Mobile Robot Navigation; Collision Free; Fuzzy Logic; Webots Simulator; E_Puck

\section{INTRODUCTION}

During the last two decades, Autonomous mobile robots have been increasingly utilized in the various field (industrial, service robotic). With the development of robot technology, robots and peripherals can become more sophisticated, reliable and miniaturized. Many problems need to be solved to do a task with a mobile robot, such that the mobile robot such as path planning and robot navigation problem. Various techniques and algorithms are developed for doing such robots usefulness. Collision-free robot navigation, trajectories with the controlling of robots' speed and direction is being focused. In order to observe the surrounding environment, different kinds of sensors can be mounted on the mobile robots. Steering the robot in the collision-free path belonged to effects of these sensors values. In detection and obstacles avoidance, as well as go to goal behavior of the mobile robot, there are many sensor techniques that have been approved to be operative. To drive a mobile robot between the starting point to the destination point, without colliding any obstacles along its way, there are many soft computing techniques used in literature. Some of the themes are; fuzzy logic, neurofuzzy, particles Swarm optimization(PSO) and genetic algorithm which are well-known algorithms. In this study, it is aimed to use a fuzzy logic controller to handle multiple infrared sensor information as input parameters and to implement a mobile robot control application without collision and moving to the target. We designed an expert system for this methodology and created appropriate decision rules for the desired output values.

The paper is organized as follows: Section 2 presents the related work. Section 3 demonstrates the proposed methodology based on the fuzzy logic system. Section 4 illustrates the simulation implementations. Section 5 discusses the results and Future work in details respectively.

\section{RELATED WORK}

Navigation of a mobile robot in a given environment with obstacles needs to be a controlled movement which is from a start position to a goal position with collision-free capabilities. Navigating in an unknown environment requires control approaches to be able to cope with ambiguity [1]. Soft computing techniques such as fuzzy logic, evolutionary and Particle Swarm Optimization (PSO) are widely used in mobile robot navigation [2]. The fuzzy logic term, one of the soft computing methods, was proposed by Lotfi A. Zadeh at the beginning of the 1990s [3], [4]. The Fuzzy controllers have certain advantages such as; simplicity of control, low-cost and the possibility to design without knowing the exact mathematical model of the process [5][6]. Fuzzy control with different types of membership functions have been designed and fuzzy control algorithms have been developed for mobile robot behavior control [7][8]. A collision avoidance mechanism for mobile robot navigating in unknown environments based on a dynamic recurrent neuro-fuzzy system (DRNFS), proposed by Chen and Richardson [9]. Multi-sensor fusion technology based collision avoidance approximation with ultrasonic and infrared sensors was used in [10], [11]. A novel sensor data methodology for mobile robots in unknown environments was conceived in [12]. In addition to this, fuzzy algorithms were used for the robot's path planning. In [13], a fuzzy logic controller was used for navigation and obstacle avoidance. In this study, the used mobile robot is equipped with eight infrared range sensors and the performances of intelligent navigation algorithm have been evaluated. Infrared range sensors data applying to the fuzzy logic control inputs, this data has been used to avoid the obstacle when moving the robot from a starting point to a destination point [14] on a map. Additionally, Robot control 
and navigation applications have been implemented using different algorithms and methods [15]- [17] [18], [19]. Webots simulator is developed for the robots which are a development environment used to model, program and simulate mobile robots. This simulation is equipped with various controls to allow the user to setup different experiments [20]. Many academic studies have been carried out using Webots software. Some of them are presented here. Pandey et al [21], designed sensor integration based fuzzy logic controller to create a collision-free path, using webots simulator to check simulation result. In [22] [23], webots and Khepera II miniature mobile robot utilized as a platform for the investigation of Neurol Q-Learning controller. Almasri et al [24] present a collision-free mobile robot navigation using fuzzy logic fusion model. They used webots simulator for modeling the environment and the robot. Webots simulator has been implemented and tested through simulation and realtime experiments. Mobile robot's movement in a cluttered area with static obstacles was controlled via fuzzy approach and used webots simulator and Matlab software. it is indicated that webots and Matlab are suitable tools to develop and simulate mobile robot navigation [25].

\section{SYSTEM OVERVIEW}

In our proposed methodology, four important criteria have been used for mobile robot collision-free navigation with the integration of fuzzy logic controller. The mobile robot equipped with several external sensors that is distance sensors, camera, and GPS. Infrared sensors' values were used for distance measurement. Robot position information obtained by using supervisor function, of webots simulator, it is used to identified robot position parameters. The supervisor capability is to move and rotate any objects in simulation environment by using Emitter and Receiver nodes and track the coordinate of that objects. The aim of the proposed method is summarized in Fig.1 and Fig2. It is shown in these figures that two fuzzy logic behaviors (Go to goal Fuzzy control (GGFC) and collision-free Fuzzy control (CFLC)) are designed and implemented for robot navigation and obstacles avoidance in an unknown environment. Fuzzy logic controller consisted of three parts: linguistic variables, membership function, and fuzzy rules. Sensors variables are inputs and wheels speed are output linguistics variables for fuzzy architecture. Go to goal behavior (GGFC) controller has two inputs which are distance and angle error between robot and goal as shown in Fig.2. Collison free behavior (CFFC)) controller has six sensors value as input which's are right, left and front infrared sensors as shown in Fig.1.

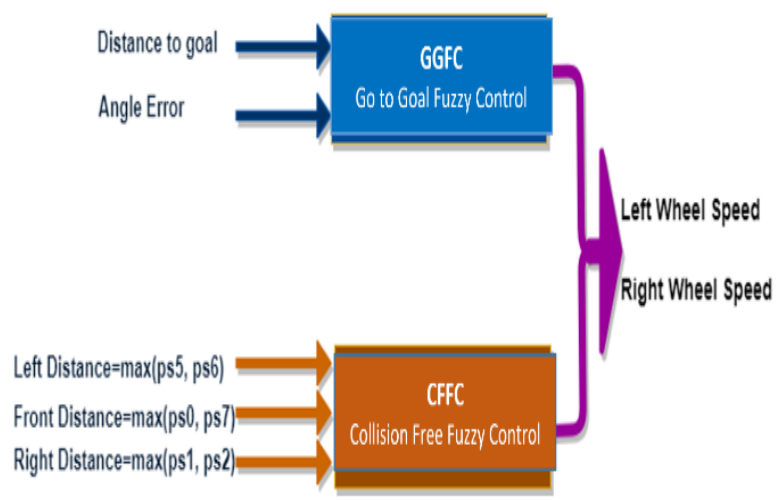

Fig.1 Fuzzy Logic Controller System

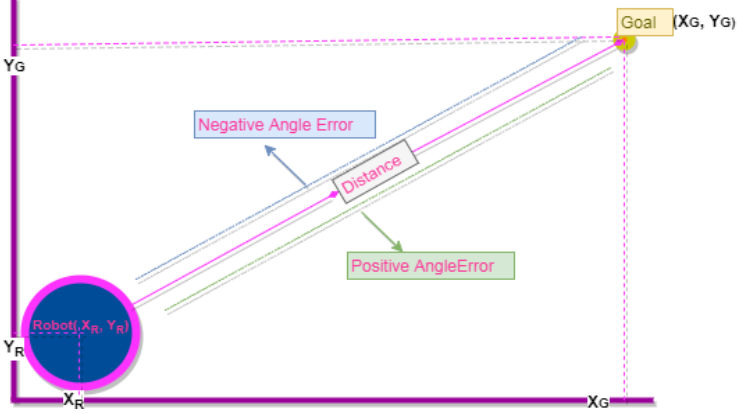

Fig.2 Robot and Goal in XY coordinate

\subsection{Modeling the Robot and Environment}

The Webots simulation software was used to create a suitable environment and robot for mobile robot simulation, as shown in Figure 3b). Webots environment included different shaped obstacles and E-Puck mobile robot in our experiments which is equipped with sensors and actuators such as camera, GPS [20]. Project file created in webots simulator composed of four main windows which are: scene tree, texts editor, world (3D simulation environment) and console windows. The differential driver E-puck robot was built using the Webots software. It is equipped with eight IR sensors in range of 0 to 2000 values, to detect obstacles around. Fig.3-a shows the Epuck configuration of IR sensors, symbolized as ps0 till ps7 and gathered as a bipartite group.

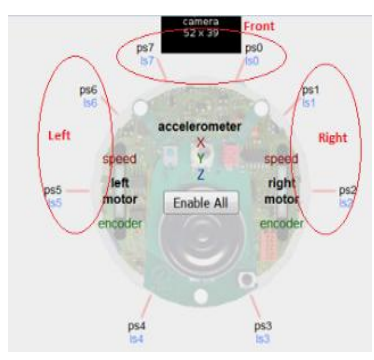

a)

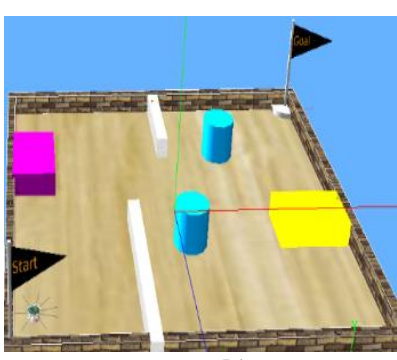

b)
Fig. 3. The top view of a) E-puck robot with various sensors b) Environment with various types of obstacles

\subsection{Fuzzy Logic Controller}

In this section, the fuzzy controller is designed and implemented for a mobile robot, at the same time navigation and obstacle avoidance task. This designation has been used six distance sensors. The fuzzy structure is composed of three main parts which are: fuzzification, inference engine, and defuzzification. The fuzzification phase occurs from linguistic variable transformed in each real value's input and outputs of fuzzy sets. The second part is a fuzzy inference that governs the fuzzy logic control process and combines the facts obtained from the defuzzification of the rule base. To transform the subset of output which is computed by the inference engine is the basic function of defuzzification block. Two fuzzy logic control methods have been used, one is Go to goal behavior (GGFC) another is collision free behavior (CFFC)) as shown in Fig.1.

\subsubsection{Go to Goal Fuzzy Controller (GGFC)}

The developed go to goal behavior controller was used two inputs (distance and angle error), and to generate the right and left wheel speed of the mobile robot move from start to goal point MATLAB Fuzzy Logic controller was used. These inputs are the distance between robot and goal, and error angle between robot and goal. The values of inputs variable were calculated by the following equations: 
$\operatorname{distance}(\mathrm{d})=\sqrt{\left(X_{G}-X_{R}\right)^{2}+\left(Y_{G}-Y_{R}\right)^{2}}$

$\phi=\theta_{T}-\theta$

$\theta_{T}=\tan ^{-1} \frac{\left(Y_{G}-Y_{R}\right)}{\left(X_{G}-X_{R}\right)}$

ğ̈

Fig.1 demonstrates the block diagram of the proposed system structure. The representation membership function distribution for GGFC behavior was graphically illustrated in Fig.4. In this experiments, three membership function are considered for inputs and four membership function are considered for outputs. The linguistic variables for distance are namely Near, Normal and Far respectively. Inputs for angle error are respectively named as Negative, Zero and Positive. Output range of this controller for wheel speeds, linguistically is namely Negative, Zero and Positive. The wheels are about 1000 steps / $\mathrm{s}$ in maximum speed that corresponds to one-wheel revolution in per second [26].

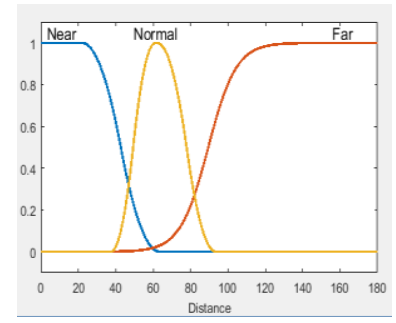

a.

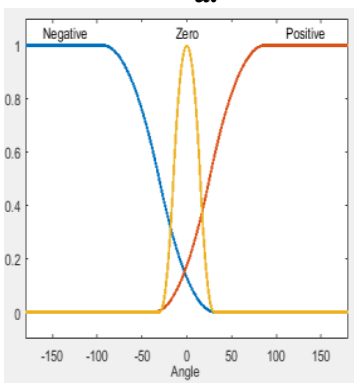

c.

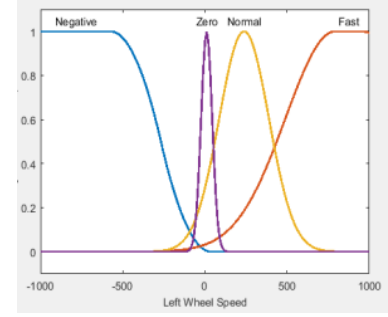

b.

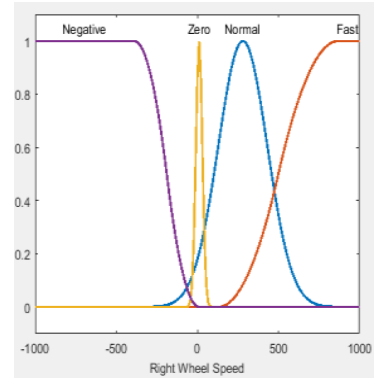

d.
Fig 4. Distribution of membership function of GGFC a, c) input variables (distance, angle), b, d) output variables (Left and Right Wheel Speed)

\subsubsection{Collision-Free Fuzzy Controller (CFFC))}

The ability of the mobile robot to navigate without collision in an environment shows that it performs intelligent control behavior. CFFC is used for the intelligently behavior of mobile robot in the environment with obstacles. The inputs of this intelligence system are distances, between robot and obstacle, which are obtained from distance sensors surrounding robot. There are eight sensor data for inputs to the CFFC and two outputs. These sensors are abbreviated as ps0...ps7 as shown in Fig. 3 a. The sensors were handled in groups of two. Six sensor values were calculated for the right, left and front. When calculating the distance, the maximum value of the sensors in the group was used. The obstacles detection amount of light in the range of 0 to about 2000 was divided into three linguistic variables; Near, Medium and Far. The dispersion of membership function for inputs variable and two outputs named as right wheel speed and left wheel speed was illustrated in the Fig.5.and Fig.6.

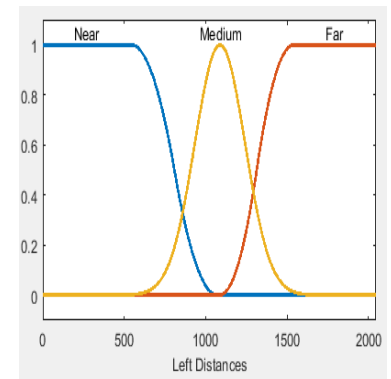

a.

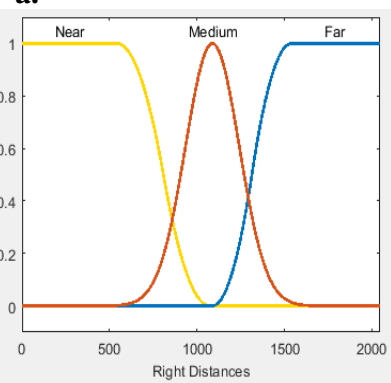

c.

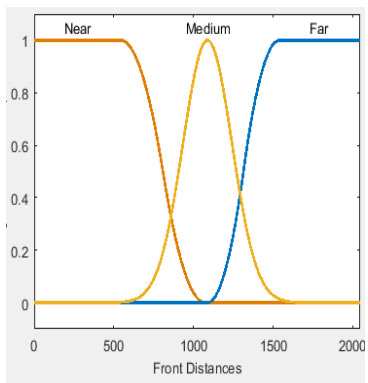

b.
Fig.5(a, b, c) Distribution of membership function for input variables of CFFC.
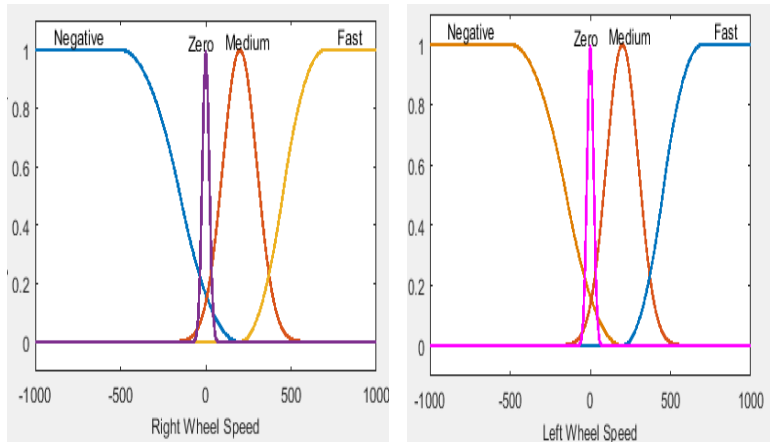

Fig.6 Distribution of membership function for output variables of CFFC

\section{SIMULATION RESULTS}

In this section, the results of the proposed approaches are simulated and the results obtained for different scenarios are shown using MATLAB software and Webots simulator. An environment, with various dynamic obstacles in different shapes and sizes, has been designed and approved by simulation. Fig.7 present snapshots of the test environment. 


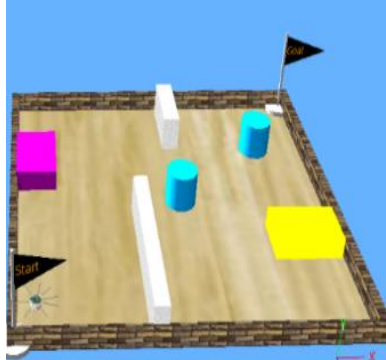

a)

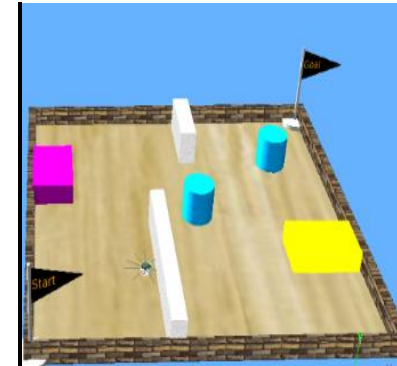

b)

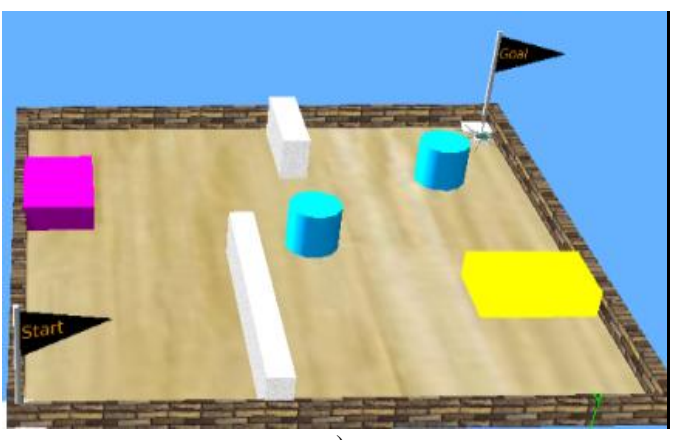

c)

Fig 7. Simulation snapshots of test environment (a)

The beginning of the moving forward; (b) The robot detects an obstacle on its way and avoids the obstacle; (c) The robot reached the goal

The robot started from an initial position $\left(\mathrm{X}_{0}, \mathrm{Y}_{0}\right)$ moving to the goal position $\left(\mathrm{X}_{\mathrm{G}}, \mathrm{Y}_{\mathrm{G}}\right)$ using GGFC and CFFC controller. Several tests for different configuration of de robot direction towards goal position have been implemented. It was considered that the goal position is static in addition to the different initial position of the mobile robot. The proposed controller has been tested with different situation. In table1 five scenarios information has been given.

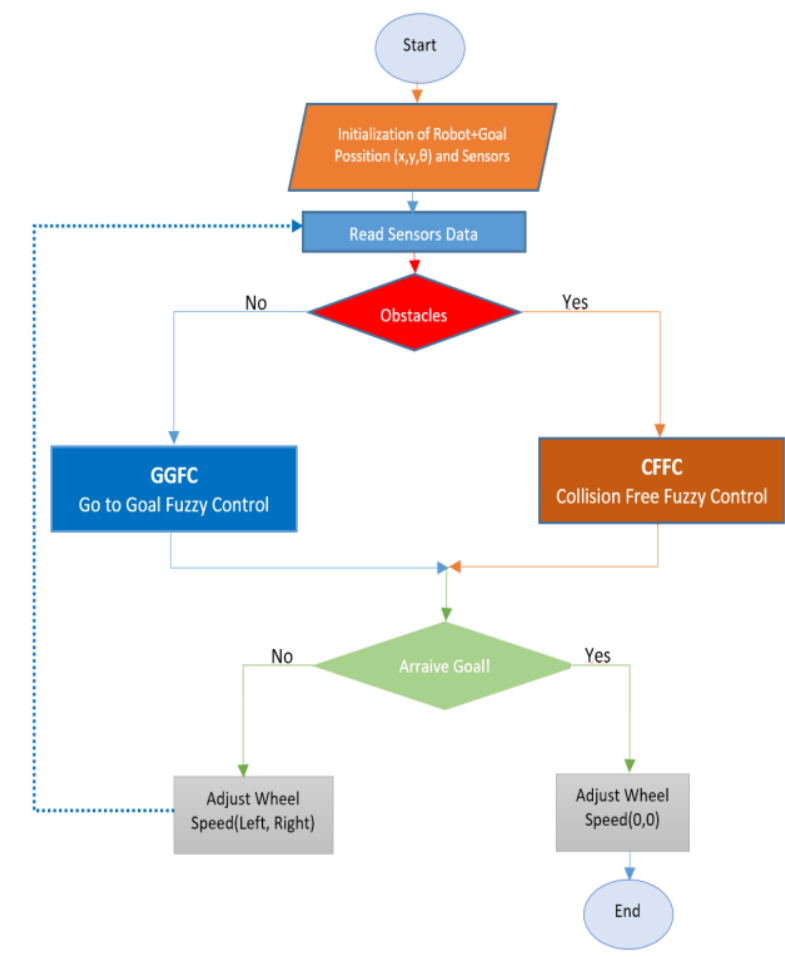

Fig.8. Flowchart of the proposed methodology

Table 1. The initial of robot and goal position, distances between robot and goal and average speeds of the robot in a safe environment

\begin{tabular}{|c|c|c|c|c|c|c|c|c|c|}
\hline \multirow{2}{*}{ : } & \multirow{2}{*}{ 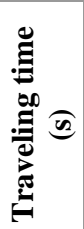 } & \multicolumn{3}{|c|}{ Robot initial position } & \multicolumn{2}{|c|}{ Goal Position } & \multirow[t]{2}{*}{ Distance(initial) } & \multicolumn{2}{|c|}{$\begin{array}{c}\text { Average Speed } \\
\text { (steps / s) }\end{array}$} \\
\hline & & $\mathrm{x}$ & $\mathrm{y}$ & Angle(Radian) & $\mathrm{x}$ & $\mathrm{y}$ & & Left & Right \\
\hline $\mathrm{S} 1$ & 600 & 0.09 & 0.19 & 0.76 & 2 & 2 & 2.63 & 318,94 & 329,51 \\
\hline $\mathrm{S} 2$ & 400 & 0.12 & 0.24 & 0.75 & 2 & 2 & 2.57 & 322,37 & 306,72 \\
\hline $\mathrm{S} 3$ & 350 & 0.10 & 0.21 & 0.72 & 2 & 2 & 2.56 & 302,36 & 319,93 \\
\hline $\mathrm{S} 4$ & 400 & 0.13 & 0.25 & 0.78 & 2 & 2 & 2.57 & 282,32 & 310,78 \\
\hline S5 & 450 & 0.07 & 0.20 & 0.75 & 2 & 2 & 2.64 & 346,42 & 323,16 \\
\hline
\end{tabular}

Table 1 shows the navigation of the mobile robot in various initial position and stabled goal position. The angular orientation and position information of the mobile robot obtained from the GPS sensor were obtained according to the webots global coordinate system information. In the proposed method, two fuzzy control architecture is used as mentioned before.
The first is the GGFC which is used to make the mobile robot go to the target, and the other is the CFFC which is used to prevent the robot from obstacles. GGFC and CFFC work together as a control switches to control the wheel speeds. The proposed methodology's flowchart is illustrated in Fig.8. 


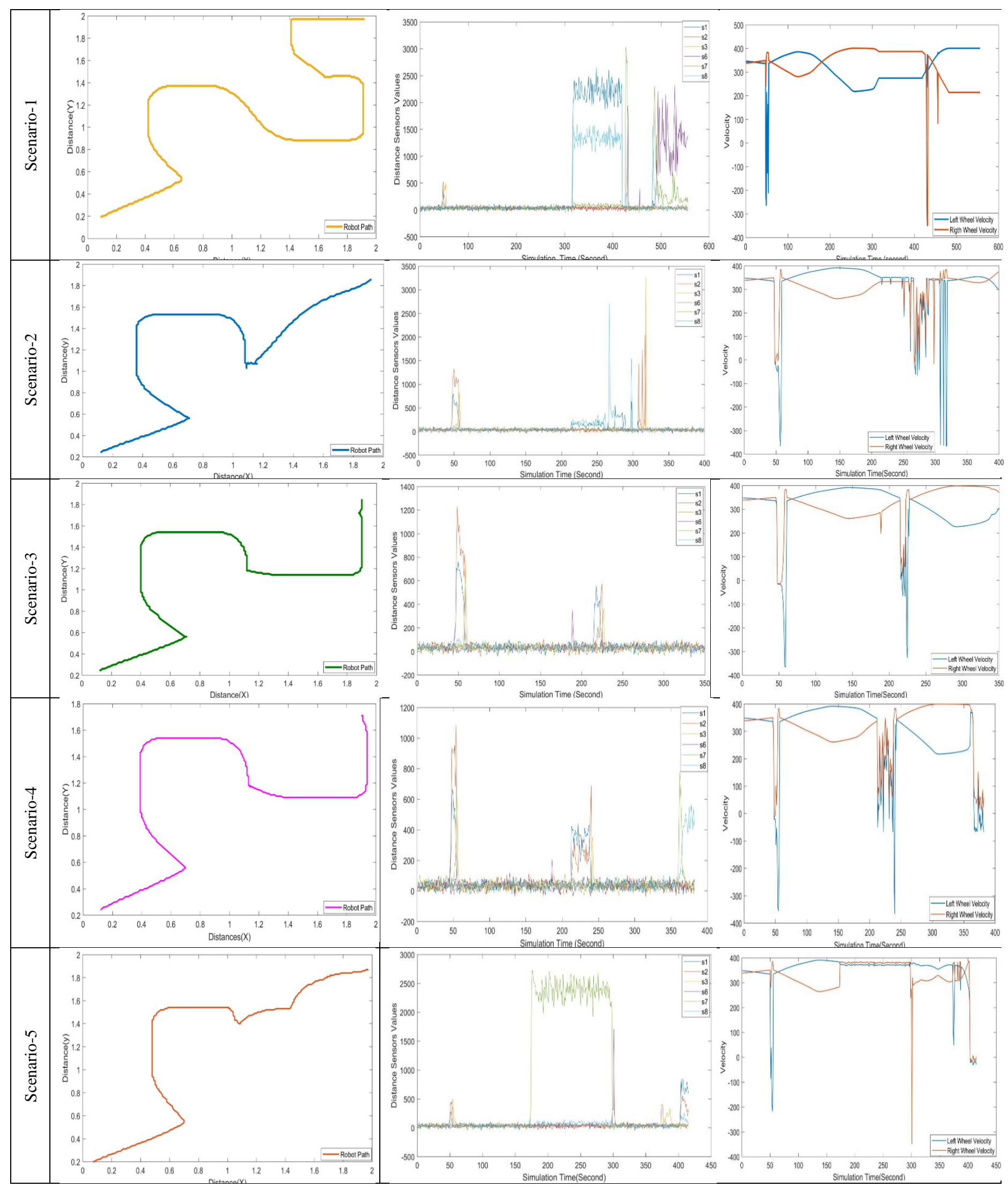

Fig. 9, Experimental results of the proposed controllers (CFFC and GGFC) for five scenarios. The first column illustrates robot path between initial and goal position. The second column illustrates six distances sensors values through simulation time and the third column illustrates the wheel velocity during robot motion in different simulation times respectively 
At the beginning of the simulation, the robot starts sensing the environment for possible obstacle detection. An automatic mechanism based on critical parameters has been proposed for the desired solution using the distance sensors consisting of six inputs. These input sensors are divided into groups of two. As can be seen in Figure 3a, the maximum values of the first (ps1) and second (ps2) sensors represent the obstacle information perceived from the right side. The maximum value of the fifth (ps5) and sixth (ps6) sensor data for the left side is used, and the maximum of the zero (ps0) and seventh (ps7) sensor data for the front side is used. The coordinates of the robot position and the target position are obtained from the GPS information system used in the Webots simulation. The direction of the robot (angle), the position according to the target and the distance between the target and the robot were calculated by Eq.1 and Eq.2. The performance of GGFC and CFFC simulations in several scenarios were illustrated in Fiq.9. Figure 9 shows a graphical representation of the change in sensor values during the robot movement. The initial value of the wheel speeds is set at 350 steps / sec. When sensors value greater than threshold values, then the control of the robot changes from the GGFC to the CFFC to avoid the obstacle as presented in Fig.9. As a result, the robot will adjust its speed accordingly to avoid colliding and go to goal using both CFFC and GGFC.

\section{CONCLUSION AND FUTURE WORK}

Navigation of mobile robot from an initial position to the goal position without collision any obstacles in an unknown environment is included many challenges. Soft computing method like FLC is operative methods in this fields. An automatic mechanism based on the critical parameter for the desired solution has been proposed using the distance sensors consisting of six inputs and two outputs. To obtain robot and target position, webots GPS was used. The proposed methodology has been implemented in the webots simulator and MATLAB software. Five different scenarios were designed and different parameter were used as shown in table 1and Fig.9. The experimental results have shown that the proposed architecture provides an efficient and flexible solution for autonomous mobile robots and the objective of this research has been successfully achieved. This research also indicated that WEBOT and MATLAB are suitable tools that could be used to develop and simulate mobile robot navigation system. We have in mind to do design and implement new optimized motion control using soft computing techniques such as graduated fuzzy type and intelligence wheelchair for disabled people to perform the navigation task in real time integrated brain-machine interface application for the future.

\section{REFERENCES}

[1] T. Rourkela, "Design and Analysis of Intelligent Navigational Controller for Mobile Robot Krishna Kant Pandey Design and Analysis of Intelligent Navigational Controller for Mobile Robot Master of Technology."

[2] M. Algabri, H. Mathkour, H. Ramdane, and M. Alsulaiman, "Comparative study of soft computing techniques for mobile robot navigation in an unknown environment," Comput. Hum. Behav., vol. 50, no. September, pp. 42-56, 2015.

[3] L. Zadeh, "Fuzzy logic, neural networks, and soft computing," Commun. ACM, vol. 37, pp. 77-84, 1994.

[4] L. A. Zadeh, "Soft computing and fuzzy logic," IEEE Softw., vol. 11, no. 6, pp. 48-56, 1994.
[5] P.-H. Lin, S. Hwang, and J. Chou, "Comparison on fuzzy logic and PID controls for a DC motor position controller," Ind. Appl. Soc. Annu. Meet. 1994 Conf. Rec. 1994 IEEE, pp. 1930-1935, 1994.

[6] Y. Tipsuwan and M.-Y. Chow, "Fuzzy Logic Microcontroller Implementation for DC Motor Speed Control," Proceedings of IEEE Industrial Electronics Society IECON'99, vol. 3. pp. 1271-1276, 1999.

[7] X. Li and B. Choi, "Design of Obstacle Avoidance System for Mobile Robot using Fuzzy Logic Systems," Int. J. Smart Home, vol. 7, no. 3, pp. 321-328, 2013.

[8] P. Rusu, E. M. Petriu, T. E. Whalen, A. Cornell, and H. J. W. Spoelder, "Behavior-based neuro-fuzzy controller for mobile robot navigation," IEEE Trans. Instrum. Meas., vol. 52, no. 4, pp. 1335-1340, 2003.

[9] C. Chen and P. Richardson, "Mobile robot obstacle avoidance using short memory: a dynamic recurrent neuro-fuzzy approach," Trans. Inst. Meas. Control 34, vol. 34, no. 2-3, pp. 148-164, 2012.

[10] C. G. Rusu, I. T. Birou, and E. Szoke, "Fuzzy based obstacle avoidance system for autonomous mobile robot," 2010 IEEE Int. Conf. Autom. Qual. Test. Robot. AQTR, no. L, pp. 1-6, 2010.

[11] K. Srinivasan and J. Gu, "Multiple Sensor Fusion in Mobile Robot Localization," 2007 Can. Conf. Electr. Comput. Eng., pp. 1207-1210, 2007.

[12] R. C. Luo, C. T. Liao, and S. C. Lin, "Multi-sensor fusion for reduced uncertainty in autonomous mobile robot docking and recharging," in 2009 IEEE/RSJ International Conference on Intelligent Robots and Systems, IROS 2009, 2009, pp. 2203-2208.

[13] H. Omrane, M. S. Masmoudi, and M. Masmoudi, "Fuzzy Logic Based Control for Autonomous Mobile," Comput. Intell. Neurosci., vol. 2016, pp. 1-10, 2016.

[14] N. K. A. Al-Sahib and A. R. Jasim, "Guiding Mobile Robot by Applying Fuzzy Approach on Sonar Sensors," Al-Khwarizmi Eng. J., vol. 6, no. 3, pp. 36-44, 2010.

[15] M. Dirik, A. F. Kocamaz, and E. Donmez, "Static path planning based on visual servoing via fuzzy logic," in 2017 25th Signal Processing and Communications Applications Conference (SIU), 2017, pp. 1-4.

[16] M. Dirik, A. F. Kocamaz, and E. Dönmez, "Vision-based decision tree controller design method sensorless application by using angle knowledge," in 2016 24th Signal Processing and Communication Application Conference (SIU), 2016, pp. 1849-1852.

[17] E. Dönmez, A. F. Kocamaz, and M. Dirik, "Robot control with graph based edge measure in real time image frames," in 2016 24th Signal Processing and Communication Application Conference (SIU), 2016, pp. 1789-1792.

[18] F. Shamsfakhr and B. Sadeghibigham, "A neural network approach to navigation of a mobile robot and obstacle avoidance in dynamic and unknown environments," vol. 253906, pp. 1629-1642, 2017.

[19] X. Li, F. Liu, J. Liu, and S. Liang, "Obstacle avoidance for mobile robot based on improved dynamic window approach," vol. 25, pp. 666-676, 2017. 
[20] "Webots: overview." [Online]. Available: https://www.cyberbotics.com/overview. [Accessed: 22Jul-2017].

[21] K. K. Pandey, M. S. Pol, and P. D. R. Parhi, "Using an AI Technique Navigation and Path Planning for Mobile Robot on Webots Platform," vol. 2014, pp. 27-31, 2014.

[22] V. Ganapathy, S. C. Yun, and W. L. D. Lui, "Utilization of webots and Khepera II as a platform for Neural QLearning controllers," 2009 IEEE Symp. Ind. Electron. Appl. ISIEA 2009 - Proc., vol. 2, pp. 783-788, 2009.

[23] V. Ganapathy, W. Lik, and D. Lui, "Application of Neural Q-Learning Controllers on the Khepera II via
Webots Software," Mech. Eng., no. December, pp. 1113, 2008.

[24] M. Almasri, K. Elleithy, and A. Alajlan, "Sensor Fusion Based Model for Collision Free Mobile Robot Navigation," Sensors, vol. 16, no. 24, pp. 1-24, 2016.

[25] M. I. Ibrahim, N. Sariff, J. Johari, and N. Buniyamin, "Mobile robot obstacle avoidance in various type of static environments using fuzzy logic approach," Electr. Electron. Syst. Eng. ICEESE 2014 Int. Conf. On, pp. 8388,2014

[26] "Webots documentation: Webots Reference Manual." [Online].Available:https://www.cyberbotics.com/doc/ref erence/index?version=8.5.[Accessed:25-Jul-2017]. 